\title{
Efficacy of brief motivational interviewing to improve adherence to inhaled corticosteroids among adult asthmatics: results from a randomized controlled pilot feasibility trial
}

This article was published in the following Dove Press journal:

Patient Preference and Adherence

10 November 2014

Number of times this article has been viewed

\author{
Kim L Lavoie ${ }^{1-3}$ \\ Gregory Moullec 1,2,4 \\ Catherine Lemiere ${ }^{2}$ \\ Lucie Blais ${ }^{2}$ \\ Manon Labrecque ${ }^{2}$ \\ Marie-France Beauchesne ${ }^{2}$ \\ Veronique Pepin ${ }^{2,4}$ \\ André Cartier ${ }^{2}$ \\ Simon L Bacon 1,2,4 \\ 'Montreal Behavioural Medicine \\ Centre, ${ }^{2}$ Research Centre, Hôpital \\ du Sacré-Cœur de Montréal - \\ A University of Montreal Affiliated \\ Hospital, Montréal, ${ }^{3}$ Department \\ of Psychology, University of Quebec \\ at Montreal (UQAM), Succursale \\ Center-Ville, Montreal, ${ }^{4}$ Department \\ of Exercise Science, Concordia \\ University, Montreal, Quebec, Canada
}

Correspondence: Kim L Lavoie Department of Psychology, University of Quebec at Montreal (UQAM), PO Box 8888, Succursale Center-Ville, Montreal, Quebec H3C 3P8, Canada

$\mathrm{Tel}+\mathrm{I} 5 \mathrm{I} 49873000$ (3835)

Fax + I $5 \mid 49877953$

Email k-lavoie@crhsc.rtss.qc.ca
Purpose: Daily adherence to inhaled corticosteroid (ICS) regimens is one of the most important factors linked to achieving optimal asthma control. Motivational interviewing (MI) is a client-centered communication style that focuses on enhancing intrinsic motivation to engage in appropriate selfmanagement behaviors. MI has been shown to improve a variety of health behaviors including medication adherence in other disorders, but its efficacy for the improvement of ICS adherence in asthmatics has yet to be examined. This pilot "proof of concept" trial assessed the feasibility of MI to improve daily ICS adherence and asthma control levels in adult asthmatics.

Methods: Fifty-four poorly controlled (Asthma Control Questionnaire [ACQ] score $\geq 1.5$ ), highly nonadherent (filled $<50 \%$ of ICS medication in the last year) adult asthmatics were recruited from the outpatient asthma clinic of a university-affiliated hospital. Participants underwent baseline assessments and were randomly assigned to MI $(3 \times 30$ minutes sessions within a 6-week period, $n=26)$ or a usual care (UC) control group $(n=28)$. ICS adherence (\% pharmacy refills) and asthma control (ACQ, Asthma Control Test [ACT]) were measured at 6 and 12 months postintervention. Mixed model repeated measure analyses for both intent-to-treat and per-protocol were used. Results were adjusted for a priori-defined covariates including baseline adherence. Patients in the MI group also reported their impressions of the intervention.

Results: Six-month adjusted intent-to-treat analyses revealed a mean change in the percentage of ICS refills of $13 \%$ in the MI group vs $6 \%$ in the UC group (between group net improvement associated with $\mathrm{MI}=+6 \%$ [-12\% to $24 \%]$ ). Twelve-month analyses revealed a mean change in the percentage of ICS refills of $11 \%$ (MI group) vs $7 \%$ in the UC group (between group net improvement associated with $\mathrm{MI}=+3 \%[-11 \%$ to $18 \%]$ ) representing an effect size (ES) of $d=0.20$ (medium). Six-month adjusted net improvement in ACQ and ACT scores between MI and control groups was -0.2 and +0.7 , respectively, with improvements being even more pronounced at 12 months ( $\mathrm{ACQ}=-0.5 ; \mathrm{ACT}=+1.1 ; \mathrm{ES}, d=0.12$ and 0.18 [small], respectively). Interestingly, 6- and 12-month net improvements in asthma self-efficacy (which is specifically targeted by MI) improved by +0.4 and +0.4 , respectively, with an ES of $d=0.46$ (marginally large) Patients in the MI group rated the intervention overwhelmingly positively in terms of usefulness, pertinence, feasibility, enjoyableness, and likelihood to change adherence behavior.

Conclusion: Results suggest that a brief(90 minutes) MI intervention that targets ICS adherence can produce clinically significant improvements in adherence behavior, asthma control levels, and asthma self-efficacy in poorly controlled nonadherent asthmatics at 6 months that are maintained for 1 year, and it is well accepted by patients. Future studies including larger sample sizes, modified intervention designs (eg, MI "booster" sessions), and assessments of the extent behavior changes translate into clinical improvements (eg, in asthma control and quality of life) are warranted.

Keywords: motivational interviewing, asthma, adherence, inhaled corticosteroids, asthma control 


\section{Introduction}

Asthma is a chronic disorder of the airways characterized by reversible airway obstruction caused by persistent inflammation, resulting in recurrent wheeze, cough, and chest tightness. Asthma represents a significant societal burden, costing over 2 billion dollars annually in Canada ${ }^{1}$ and 56 billion annually in the United States. ${ }^{2}$ The high burden of asthma, which affects 300 million people worldwide, appears to be related to poor asthma control, which is associated with more frequent asthma symptoms, worse pulmonary function, increased health service use, and increased functional impairment. ${ }^{3-5}$

The availability of effective treatments suggests that asthma can be well controlled in most patients, ${ }^{6}$ yet the vast majority of asthmatics remain poorly controlled. ${ }^{3,4}$ Achieving optimal asthma control relies upon several behavioral factors, including minimizing exposure to known triggers (eg, pets, dust) and engaging in appropriate health behaviors (eg, avoiding tobacco smoking and adhering to medication). ${ }^{7}$ According to current asthma treatment guidelines, ${ }^{5}$ daily adherence to inhaled corticosteroid (ICS) regimens is one of the most important behavioral factors linked to achieving optimal asthma control, because it has been shown to be effective for achieving good asthma control even in patients who fail to adequately minimize exposure to known triggers (eg, indoor allergens, exercise). Despite the efficacy of ICSs, recent trends suggest that asthmatics are not adherent to these medications, with rates being as low as $32 \%$, even in patients with severe asthma. ${ }^{8-10}$ Explanations for this range from simply forgetting (nonintentional adherence) to having distorted illness and medication beliefs (intentional nonadherence). ${ }^{11-14}$

Currently, there is a paucity of research on interventions specifically designed to improve ICS adherence among adult asthmatics. Most previous work has been conducted in pediatric samples, ${ }^{15-18}$ which has yielded inconsistent results that are difficult to generalize to adult samples because pediatric adherence often relies upon parental beliefs, attitudes, and behavior. Of the studies conducted among adults, ${ }^{19-29}$ most suffer from important methodological limitations (eg, no objective measure of adherence, ${ }^{22,24}$ failing to conduct intent-to-treat (ITT) analyses ${ }^{19-26}$ as per the CONSORT statement ${ }^{30}$ that make them difficult to generalize or interpret with confidence. Moreover, the majority of interventions to date have used primarily educational approaches, which have been the subject of much criticism, as several studies have shown that there is little association between patient's knowledge about a disease and its treatment, and their actual treatment behavior., ${ }^{9,31-33}$ This may be due to the fact that most education-based approaches do not address motivation and self-efficacy to engage in a particular behavior, both of which are key to behavioral intention and action. ${ }^{34}$

Motivational interviewing (MI) is a client-centered intervention that focuses on enhancing (1) intrinsic motivation to engage in a particular behavior and (2) an individual's level of self-efficacy or confidence in their ability to engage in that behavior. ${ }^{35}$ Brief MI sessions (eg, 1-5, 15-30-minute sessions) have been shown to improve a variety of health behaviors (eg, reduce alcohol consumption, improve dietary habits, increase exercise behavior, and improve medication adherence) and health outcomes (reduce blood pressure, body mass index, and cholesterol levels). ${ }^{36-38}$ However, no studies to date have assessed the efficacy of using MI to improve ICS adherence in asthmatics.

The purpose of this study was to conduct a single-site randomized controlled pilot trial to assess the feasibility of brief MI to improve daily ICS adherence and asthma control levels in a sample of poorly controlled, nonadherent asthmatics in a tertiary care setting. We expected to observe improved rates of ICS adherence (measured using pharmacy refill rates) and asthma control at 6 and 12 months postintervention among patients randomized to the MI intervention compared with those randomized to a usual care (UC) control condition.

\section{Methods}

\section{Participants}

A total of 54 adult asthma patients meeting eligibility criteria and agreeing to participate were recruited from the outpatient clinic of Hôpital du Sacré-Coeur de Montréal (HSCM) by a trained research assistant between June 2008 and September 2010. Patients were included if they were 18 years and older, had a primary diagnosis of moderate-severe persistent asthma (confirmed by chart evidence of a $20 \%$ fall in forced expiratory volume in one second $\left[\mathrm{FEV}_{1}\right]$ after methacholine challenge and/or bronchodilator reversibility in $\mathrm{FEV}_{1}$ of $\geq 20 \%$ predicted $^{39}$ as per Global Initiative for Asthma guidelines), ${ }^{5}$ and had been prescribed a stable dose of ICS medication (minimum prescribed dose of $250 \mu \mathrm{g}$ fluticasone equivalent per day) for at least 12 consecutive months prior to study entry. Patients had to have been identified as having uncontrolled asthma (Asthma Control Questionnaire [ACQ] score $\geq 1.5$ ) and nonadherent to ICS medication based on having filled less than $50 \%$ of their prescription for ICSs over the last year. Patients were excluded if they had a comorbid 
medical condition that conferred greater risk for morbidity than asthma (eg, chronic obstructive pulmonary disease, cardiovascular disease), if they had severe psychopathology (eg, schizophrenia), current substance abuse, an apparent cognitive or language deficit, or if they were or planned to become pregnant or move outside of Quebec over the course of the study.

See Figure 1 for details regarding the flow of participants through the study. Briefly, a total of 1,530 patients presented to the outpatient asthma clinic over the course of the recruitment period, of which 1,446 patients were prescreened for inclusion. A total of 528 patients had confirmed asthma without language and cognitive deficits and were approached to participate. Of these, 185 patients (35\%) refused, resulting in a sample of 343 patients who underwent screening evaluations. Of them 289 patients did not meet the eligibility criteria, yielding 54 eligible patients who were randomized to MI or UC. This project was approved by the Human Ethics Committee of HSCM, and all patients provided written informed consent.

\section{Study design}

This study was a single-site randomized controlled pilot "proof of concept" trial designed to test the feasibility of

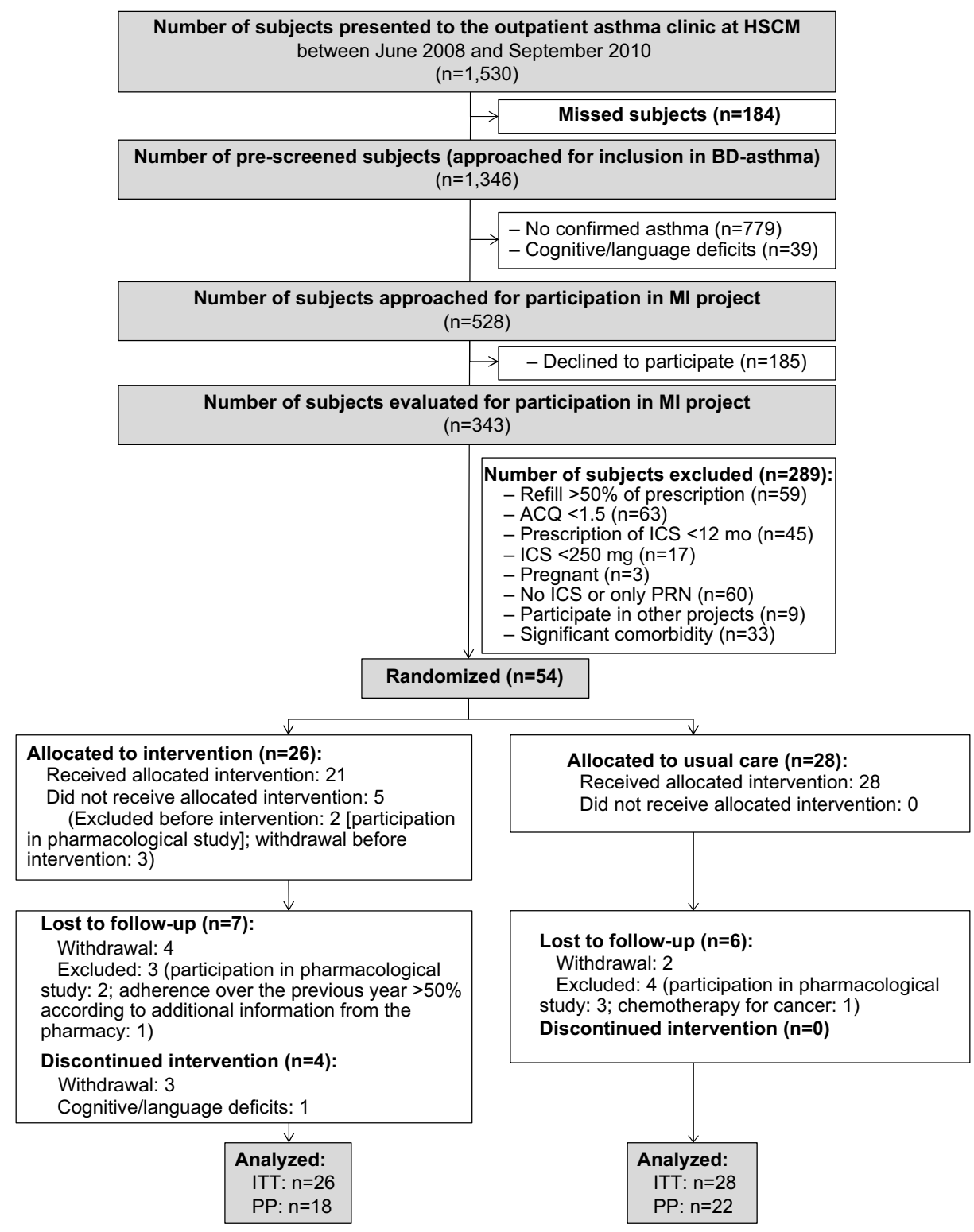

Figure I Study flow chart.

Abbreviations: HSCM, Hôpital du Sacré-Coeur de Montréal; BD, twice-daily; MI, motivational interviewing; ACQ, Asthma Control Questionnaire; ICS, inhaled corticosteroid; PRN, as needed; ITT, intent-to-treat; PP, per-protocol; mo, months. 
brief MI vs UC on ICS adherence among poorly controlled, nonadherent adult asthmatics. This pilot trial was conducted in accordance with the National Institute of Health's Obesity Related Behavioral Intervention Trials (ORBIT) guidelines on the development of behavioral interventions, which emphasize using a phasic approach that mirrors drug development in the pharmaceutical industry. ${ }^{40}$ According to ORBIT, this trial could be characterized as a Phase IIb trial, which are smaller trials designed to determine the effect size (ES) of an intervention and its acceptability in the target population.

Patients were recruited from the outpatient asthma clinic at HSCM. Patients underwent a screening assessment including a brief sociodemographic characteristics, medical history, and psychiatric interview, as well as pulmonary function testing, on the day of their asthma clinic visit. Patients meeting all eligibility criteria were invited to participate in an intervention study designed to help patients gain better control of their asthma. They were informed that we would be collecting pharmacy refill data but not that it was the primary outcome of interest. Consenting patients returned to the hospital within approximately 4 weeks to undergo baseline assessments with the study research assistant, which included completing a battery of self-report questionnaires and conducting pulmonary function testing (standard spirometry) (see "Measures" section for details). Following the completion of all baseline assessments, patients were randomized to MI or UC using a computer algorithm that generated a random code that was kept in a concealed envelope until opened by the study coordinator at the time of randomization as per the CONSORT guidelines. ${ }^{41}$ Patients randomized to MI were told that they would meet an "asthma educator", who was a clinical psychologist with extensive MI training, for three to four (range 0-4) years, for 30-minute (range 11-61 minutes) sessions over a 4- to 6-week period. Patients randomized to UC were free to accept whatever treatments were offered to them by their treating physicians, but had no contact with the research team until completing postintervention assessments. With the exception of the visual analog scales assessing patients' impressions on the MI intervention (which were completed at baseline and immediately postintervention only), all postintervention assessments were completed in-hospital at 6 and 12 months postintervention by a research assistant who was blinded to patient group. To increase the success of blinding, patients were instructed not to disclose their group assignment to the research assistant.

\section{MI intervention}

Patients randomized to MI underwent three to four individual 15-30 minute sessions over a 4-6 week period. The educator and the patient decided together that more than three (up to a maximum of five) sessions were needed to achieve therapeutic goals. In preparation for this pilot trial, an MI intervention manual that was adapted from Rollnick et $\mathrm{al}^{34}$ for use with nonadherent asthmatics was developed. Data from focus groups including asthma patients, pulmonologists, and allied health professionals who treat asthmatics (eg, nurses, inhalotherapists) were incorporated to inform aspects of the intervention design and manual, including dose (session length, intervention duration), environment (where the intervention would be delivered and by whom), and format (individual/group, in-person/telephone) that was deemed most acceptable to key stakeholders (ie, patients, physicians, and allied health professionals who treat asthmatics). ${ }^{42}$

The delivery of the intervention adhered to the approaches, techniques, and strategies outlined in the manual to ensure intervention fidelity. These included exploring ambivalence, supporting self-efficacy, "rolling with resistance," and eliciting "change-talk." ${ }^{34}$ The overall goal of the intervention was to enhance patients' intrinsic motivation to take their ICS medication as prescribed by exploring their ambivalence about taking their medication in a way that promotes change. This is accomplished by eliciting "change-talk", which encourages patients to verbalize potential benefits of taking their medication (and the potential disadvantages of not taking their medication), and by getting patients to generate potential solutions to perceived barriers to taking their medication as prescribed. Agenda setting for each session was collaborative, and the educator took the patient's stage of change ${ }^{43}$ into account when delivering the MI intervention.

\section{$\mathrm{Ml}$ intervention integrity and quality control}

The psychologist who delivered the MI intervention had over 10 years of clinical experience and received additional MI training (2-day intensive MI workshop with interactive feedback) from an accredited MI Network Trainer, as well as specific training on asthma control strategies and use of the manual. Additionally, the psychologist delivering the intervention was supervised (via biweekly meetings and as-needed support and feedback) by the study lead investigator (KLL), a $\mathrm{PhD}$-level clinical psychologist with extensive experience in both delivering MI techniques and training other health professionals $(>6,000)$ on the use of MI in clinical settings. Finally, all intervention sessions were audiotaped, and the 
number of sessions and total session time were recorded. In order to verify treatment integrity, $10 \%$ of all sessions were evaluated by an independent expert (experienced clinical psychologist trained in use of MI) using the Motivational Interviewing Skill Code. ${ }^{44}$

\section{Usual care}

Patients assigned to UC received whatever treatments their attending physician prescribed, which may have included a prescription for ICS + reliever medication as needed, and/ or an action plan for exacerbations, and/or referral to asthma education. In order to ensure equity to all patients and reduce negative expectancy effects among patients randomized to UC (if they preferred to be randomized to the active intervention), patients assigned to UC were given the opportunity to participate in the MI intervention once they had completed the 12-month assessment (although results were not included in final analyses).

\section{Measures}

All measures were taken at baseline and at 6 and 12 months postintervention.

\section{Primary outcome: ICS adherence}

Baseline ICS adherence measures were taken for the 12-month period prior to study entry, and posttreatment measures were taken at 6 and 12 months postintervention. ICS adherence (primary outcome) was measured as a proportion according to the following equation: the number of treatment (prescription) days/182.5 days (6 months adherence) and 365.5 days (12 months adherence), respectively. Data were obtained by accessing the patients' pharmacologic chart at their community pharmacy.

\section{Secondary outcomes: self-reported adherence, asthma control, asthma-related quality of life, and asthma-related self-efficacy}

Self-reported medication adherence was measured using the Morisky scale. ${ }^{45}$ This questionnaire includes four items that are rated on a 5-point Likert-type scale, where 5= never and $1=$ very often. A sample item includes: "Some people forget to take their inhaled corticosteroid medications. How often does this happen to you?" This scale has good to excellent psychometric properties, including good reliability ( $\alpha=0.61)$ and excellent concurrent and predictive validity. ${ }^{45,46}$

Asthma control was measured using the $\mathrm{ACQ}^{47}$ and the Asthma Control Test (ACT). ${ }^{48}$ The ACQ evaluates levels of asthma control according to the standard criteria specified by the international guidelines. ${ }^{49}$ Respondents are asked to recall their symptoms (shortness of breath, wheezing, waking dyspnea, and nocturnal dyspnea), activity limitations, and bronchodilator use in the last week. One additional question assessing spirometry results $\left(\mathrm{FEV}_{1}, \%\right.$ predicted $)$ is completed by the research assistant. The ACQ contains seven items rated on a 7 -point scale $(0=$ good control, $6=$ poor control $)$ to yield a mean score out of 6 . The ACQ has demonstrated excellent measurement properties, including high intraclass correlation coefficients between 0.90 and 0.95 and good construct, cross-sectional, and longitudinal validity. ${ }^{47,50}$ A score of $\geq 1.5$ on this questionnaire is indicative of poor asthma control, ${ }^{51}$ and scores of differences or changes of $\geq 0.50$ are considered clinically significant. ${ }^{52}$ The ACT assesses the five asthma control criteria (shortness of breath, general asthma symptoms, bronchodilator use, effect of asthma on daily functioning, and overall control) on a 5-point Likerttype scale $(1=$ all the time, $5=$ not at all for symptoms and activities; $1=$ not at all controlled, $5=$ completely controlled for overall control). Scores range from 5 (poor control) to 25 (complete control) with higher scores representing better control. It has demonstrated good psychometric properties, with a test-retest reliability of 0.77 and an internal consistency of $\alpha=0.84-0.85$ (cross-sectionally) and 0.79 (longitudinally). A score of $>19$ indicates well-controlled asthma and 3 points between two groups or for changes over time are considered clinically significant..$^{53}$

Asthma-related quality of life was measured using the Asthma Quality of Life Questionnaire (AQLQ), ${ }^{54}$ which evaluates asthma quality of life across four life domains that may be negatively impacted by asthma: activity limitation (how asthma limits ability to carry out daily activities), symptoms (nature and frequency of asthma symptoms such as wheezing, shortness of breath), emotional distress (how emotionally stressful the disease is), and environmental stimuli (how difficult it is for the patient to manage or avoid environmental triggers of asthma, such as pollution, smoke, dust). It contains 32 items rated on a 7-point scale ( $1=$ maximal impairment, $7=$ no impairment) to yield a mean score out of 7. It has demonstrated excellent measurement properties, including internal consistencies of 0.81 (activities subscale) to 0.95 (overall score). ${ }^{55}$ Scores differences or changes of $\geq 0.50$ are considered clinically significant. ${ }^{56}$

Asthma-related self-efficacy was measured using the Asthma Self-Efficacy Scale. This scale evaluates how confident asthmatics are in their ability to control asthma symptoms in a variety of situations. It contains 80 items rated 
on a 4-point Likert-type scale from $1=$ not at all confident to $4=$ very confident. It has good measurement properties ${ }^{57}$ and has been successfully used in our laboratory in previous studies. ${ }^{58,59}$

\section{Patient impressions of the $\mathrm{Ml}$ intervention}

In order to assess patients' acceptability of the intervention, patients' impressions of the MI intervention were assessed pre- and postintervention using a visual analog scale $(0=$ not at all to $10=$ extremely). Patients rated the perceived usefulness, pertinence, feasibility, enjoyableness, and expected (pre) vs actual (post) likelihood of intervention to change medication adherence behavior.

\section{Statistical analyses Imputation of missing data}

Multiple imputation is recommended for studies with missing values that affect less than $60 \%$ of the sample. ${ }^{60}$ In the present study, 14 patients $(26 \%)$ had at least some missing data, so multiple imputation procedures were used. The imputation method used assumes that missing data are random, and includes all covariates that were included in the final analysis. We imputed data on cases who had missing data, producing five independent replicates of datasets. Finally, each filled-in dataset comprised 54 patients, which was the final sample size used for analyses in this study. Estimation for model coefficients was produced using PROC MINIANALYZE, which averages all estimates and adjusts standard errors according to Rubin's rule. ${ }^{61}$ Details of the amount of missing data per variable are included in Table 1. We used the PROC MI method of multiple multivariate imputation in SAS version 9.3 (SAS Institute Inc., Cary, NC, USA).

\section{Main analyses}

All analyses for this pilot study were ITT as per the CONSORT statement. ${ }^{41}$ Mixed-model approach (PROC MIXED) was used for the analysis of repeated measurements with subjects as random effect. Fixed effects included in the model were: sex, age, total ICS dose (as an indicator of asthma severity), smoking status, randomization group (MI vs UC), time, and the product term of time and group. All covariates were determined a priori based on established associations with the dependent variables. These models were used to assess the effects of the intervention on primary (\% ICS refill rates) and secondary (asthma control, asthma-related quality of life, and asthma-related selfefficacy) outcomes. Additional per-protocol (PP) analyses were done, including patients who completed the trial as planned. In addition, the ES $(d)$ was calculated within each treatment condition, and then the control group ES was subtracted from the MI group ES to compute the overall ES. The ES for each treatment condition was defined as the baseline - 12-month change in mean score divided by the standard deviation of change score. The ES was categorized as small $(<0.20)$, medium $(0.20-0.50)$, large $(0.50-0.80)$, or very large $(\geq 0.80){ }^{62}$

\section{Supplementary analyses}

Considering an increase of $15 \%$ in ICS adherence as clinically significant, we compared the percentage of patients reaching at least this change at 12 months in each group. The percentage of patients who reported as having a not well-controlled asthma (ACQ score of 1.5 or above) was also compared between MI and UC groups at 12 months.

All tests were two sided and significance level was set at $P<0.05$. Data analysis was performed using SAS version 9.3 .

\section{Results}

\section{Sample characteristics}

Demographic and medical/asthma history characteristics of the 54 participants as a function of treatment condition are presented in Table 1 and are representative of a tertiary care sample of moderate to severe asthmatics. At entry, patients from both groups showed similar characteristics except for waist circumference, which was significantly higher in UC patients $(P=0.008$; see Table S1). Baseline characteristics of the 14 patients who withdrew or were excluded from the study were also similar to those of patients who completed the trial ( $n=40$; see Table $\mathrm{S} 1)$.

\section{Primary outcome}

After adjusting for covariates, the ITT 6-month analyses revealed a mean change in \% ICS refills of $13 \%$ in the MI group vs $6 \%$ in the UC group (net improvement associated with $\mathrm{MI}=+6 \%$ [95\% CI, $-12 \%$ to $24 \%]$ ) (Table 2 ). Twelvemonth analyses revealed a mean change in $\%$ ICS refills of $11 \%$ (MI group) vs 7\% in the UC group (net improvement associated with $\mathrm{MI}=+3 \%[-11 \%$ to $18 \%]$ ) representing a medium ES $(d=0.20)$. The PP analyses revealed a comparable medium ES at 12 months $(d=0.23)$, but with larger mean changes in both groups (MI, $+16 \%$; UC, $+7 \%$ ) (Table 3 ). At 12 months, an increase of $15 \%$ or more in $\%$ ICS refills was observed in 39\% (10/26) and 36\% (10/28) of MI and UC patients (ITT, $\chi^{2}=0.04, P=0.83$ ), respectively. Among 
Table I Participants' characteristics at baseline

\begin{tabular}{|c|c|c|c|c|}
\hline & Total $(n=54)$ & $M I(n=26)$ & Control $(n=28)$ & Missing data, $n$ \\
\hline \multicolumn{5}{|l|}{ Sociodemographics } \\
\hline Age, years & $50(16)$ & $52(15)$ & $49(16)$ & - \\
\hline Men, n (\%) & $21(39)$ & $13(50)$ & $8(29)$ & - \\
\hline Cohabitation (yes), n (\%) & $30(56)$ & $16(62)$ & $14(50)$ & - \\
\hline Employed (yes), n (\%) & $27(50)$ & $13(50)$ & $14(50)$ & - \\
\hline Education level ( $\geq 12$ years), $n(\%)$ & $30(56)$ & $12(46)$ & $18(64)$ & - \\
\hline \multicolumn{5}{|l|}{ Clinical and functional profile } \\
\hline Refill: $\beta 2 L A+I C S$ or ICS alone, $n$ (\%) & $21(39)$ & II (42) & $10(36)$ & - \\
\hline Refill: combined $\beta 2 L A+I C S, n$ (\%) & $33(6 \mathrm{I})$ & $15(58)$ & $18(64)$ & - \\
\hline Total dose of combined $\beta 2 \mathrm{LA}$ and/or ICS, mg & $723(501)$ & $705(585)$ & $740(4 \mid 9)$ & - \\
\hline Anti-leukotrienes, n (\%) & $12(22)$ & $5(19)$ & $7(25)$ & - \\
\hline Anti-rhinitics, n (\%) & $19(35)$ & $8(31)$ & II (39) & - \\
\hline $\mathrm{FEV}_{1}, \mathrm{~L}$ & $2.1(0.8)$ & $2.1(0.8)$ & $2.0(0.9)$ & 3 \\
\hline $\mathrm{FEV}_{1}, \%$ predicted. & $69(24)$ & 7I (30) & $67(15)$ & 3 \\
\hline FVC, L & $2.5(1.1)$ & $2.5(1.0)$ & $2.5(1.3)$ & 3 \\
\hline FVC, \% predicted & $70(32)$ & $72(40)$ & $67(20)$ & 3 \\
\hline $\mathrm{FEV}_{1} / \mathrm{FVC}, \%$ predicted & $102(17)$ & $102(15)$ & $103(18)$ & 3 \\
\hline Atopic, $\mathrm{n}(\%)$ & $43(80)$ & $19(73)$ & $24(86)$ & - \\
\hline Current smoker (yes), n (\%) & $7(13)$ & $4(15)$ & $3(11)$ & - \\
\hline $\mathrm{BMI}, \mathrm{kg} \cdot \mathrm{m}^{-2}$ & $31(7)$ & $29(5)$ & $32(9)$ & - \\
\hline Waist circumference, $\mathrm{cm}$ & $95(15)$ & $100(18)$ & $89(9)$ & 1 \\
\hline Asthma duration, years & $24(18)$ & $21(17)$ & $26(20)$ & - \\
\hline Bronchodilator use, number last week & $7(12)$ & $9(14)$ & $6(10)$ & 4 \\
\hline ACT scores (5-25) & $17(4)$ & $17(4)$ & $17(4)$ & I \\
\hline ACQ scores $(0-6)$ & $1.9(1.0)$ & $\mathrm{I} .7(0.9)$ & $2.1(I . I)$ & 1 \\
\hline ASES total scores $(I-5)$ & $2.6(0.8)$ & $2.6(0.9)$ & $2.6(0.7)$ & 1 \\
\hline Morisky scores (5-30) & $22(5)$ & $22(5)$ & $22(5)$ & 1 \\
\hline AQLQ scores (I-7) & & & & 1 \\
\hline Total & $4.9(1.0)$ & $4.9(0.9)$ & $4.8(1.1)$ & \\
\hline Activity limitations & $4.5(1.2)$ & $4.6(1.0)$ & $4.5(1.4)$ & \\
\hline Symptoms & $5.0(1.0)$ & $5.1(1.0)$ & $4.9(1.1)$ & \\
\hline Emotional function & $5.4(1.2)$ & $5.3(1.2)$ & $5.4(1.2)$ & \\
\hline Exposure & $4.7(1.4)$ & $4.7(1.3)$ & $4.7(1.6)$ & \\
\hline
\end{tabular}

Notes: Data are expressed as mean (SD) or $\mathrm{n}(\%)$.

Abbreviations: $\mathrm{MI}$, motivational interviewing; $\beta 2 \mathrm{LA}$, long-acting beta2-agonists; ICS, inhaled corticosteroid; FEV , forced expiratory volume in I second; FVC, forced vital capacity; BMI, body mass index; AQLQ, asthma quality-of-life questionnaire; ACT, Asthma Control Test; ACQ, Asthma Control Questionnaire; ASES, Asthma Self-Efficacy Scale.

the completers, $50 \%(9 / 18)$ and $36 \%(8 / 22)$ of MI and UC patients at 12 months $\left(\chi^{2}=0.75, P=0.38\right)$, respectively, showed this change.

\section{Secondary outcomes}

Small to medium ES were obtained in change of Morisky scale scores at 12 months according to both ITT and PP analyses $(d=0.14$ [small ES] and 0.23 [medium ES], respectively).

Concerning the control of asthma symptoms, the results of the ACQ and ACT analyses did not reveal any statistically significant differences in the 12 months mean change scores between the two groups according to both ITT and PP analyses, with small ES (ACQ $d=0.12-0.13$; ACT $d=0.15-0.18$ ) (Tables 2 and 3). At 12 months, 42\% (11/26) and 61\% (17/28) of MI and UC patients, respectively, reported having poorly controlled asthma (ACQ score $\geq 1.5$; ITT, $\chi^{2}=1.83, P=0.18$ ). Among the completers, 61\% (11/18) and 77\% (17/22) of MI and UC patients at 12 months $\left(\chi^{2}=1.23, P=0.27\right)$, respectively, reported poorly controlled asthma.

Likewise, both the ITT and PP analyses failed to show any statistically significant between-group differences in the Symptom domain scores of the AQLQ (as well as a very small ES, $d=0.03$, see Tables 2 and 3). However, while ITT analyses also failed to show any significant differences in the other subscale scores of the AQLQ (ie, Activity Limitation, Environmental Impact, and Emotional domains; Table 2), the PP analyses revealed medium ESs (ranging from $d=0.31$ to 0.36) in favor of the intervention (Table 3).

Finally, a medium to large ES was obtained for the change in the total score of the asthma-related self-efficacy scale at 12 months (ITT, $d=0.43$; PP, $d=0.53$ ). 


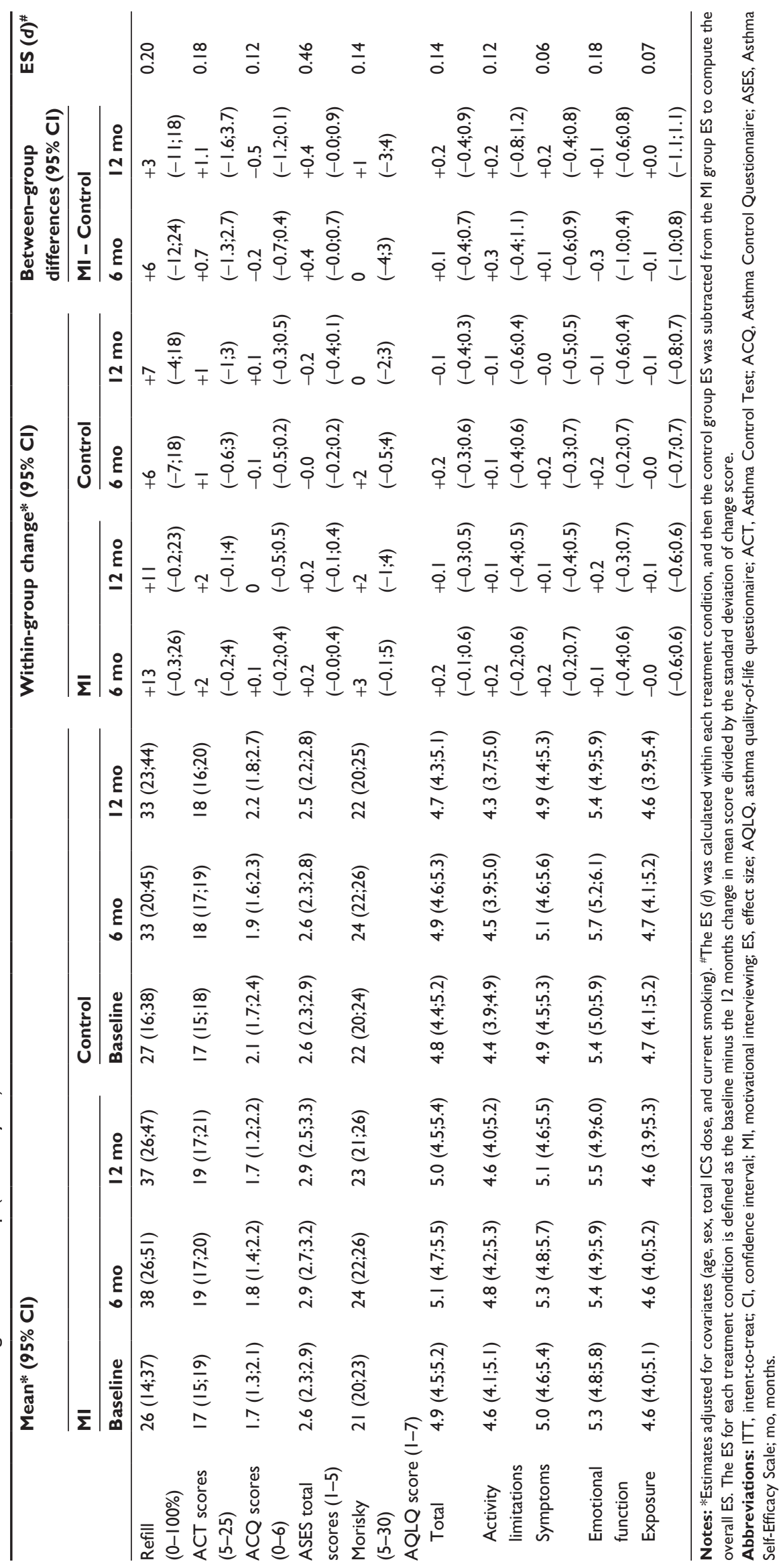




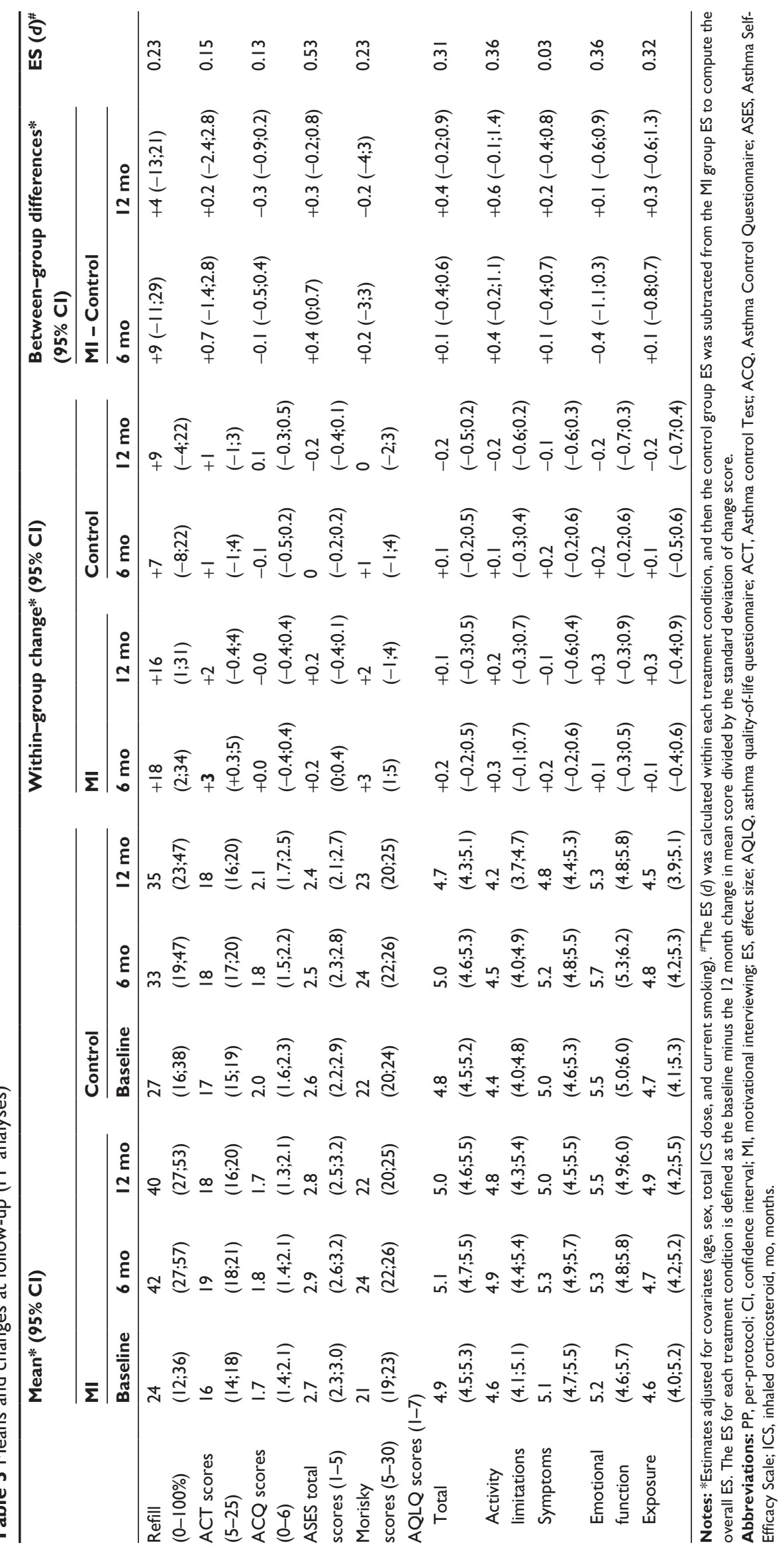




\section{Patients' impressions of the intervention}

Patients in the MI group rated (on a scale from 0 to 10) the intervention overwhelmingly positively in terms of usefulness (mean $=9.1, \mathrm{SD}$ [standard deviation] $=0.8$ ), pertinence ( mean $=8.9, \mathrm{SD}=1.2$ ), feasibility (mean $=9.2, \mathrm{SD}=0.8$ ), enjoyableness (mean $=9.5, \mathrm{SD}=0.7$ ), and likelihood of changing adherence behavior (mean $=9.2, \mathrm{SD}=1.3$ ). These ratings indicate that the intervention was very well received by patients and shows promise in terms of feasibility and effectiveness.

\section{MI treatment integrity}

MI treatment integrity was assessed by an independent rater (clinical psychologist trained in use of MI) using the Motivational Interviewing Skill Code. ${ }^{44}$ This analysis revealed Global Therapist Ratings of 6.5 (expert level), Ratio of Reflections to Questions of 1.04 (proficiency level), \% Open Questions of 38\% (below proficiency of $>50$ ), \% Complex Reflections of $61 \%$ (expert level), $\%$ MI-Consistent Responses of 95\% (expert level), \% MIInconsistent Responses of $2.6 \%$ (expert level), \% Therapist Talk Time of 37\% (expert level), and Client Change Talk Time of $71 \%$ (expert level). With the exception of $\%$ Open Questions, an evaluation of MI treatment integrity indicates that the intervention was delivered as intended, and for the most part, at the expert level.

\section{Discussion}

This pilot study assessed the feasibility of a relatively brief (90 minutes) MI intervention to improve daily ICS adherence in 54, nonadherent, poorly controlled adult asthmatics. Results of ITT analyses revealed that after adjustment for covariates, MI yielded a net improvement of $+6 \%$ in ICS adherence at 6 months and of $+3 \%$ at 12 months when compared to UC, which was not statistically significant but represents a medium ES $(d=0.20)$. A comparable ES was observed in PP (completer) analyses $(d=0.23)$ but with larger overall net improvements in ICS adherence at $6(+11 \%)$ and $12(+7 \%)$ months associated with MI relative to UC. To our knowledge, this is the first study to directly assess the feasibility of a brief MI intervention to improve ICS adherence in adult asthmatics. Although one previous study demonstrated the efficacy of MI to improve readiness to adhere to asthma medication and attitudes about adherence, it did not assess the impact of MI on actual adherence behavior per se. ${ }^{63}$ Similarly, despite observing a positive effect of MI on caregiver-reported adherence among innercity adolescents with asthma,${ }^{64}$ that study was not randomized and did not measure actual adherence, which makes it difficult to compare the results to those observed in the present study. Nonetheless, the ESs obtained in the present study are generally consistent with those reported in previous medication adherence trials conducted among patients with other medical conditions (eg, HIV). ${ }^{38}$

Of particular note is that medium effects were observed with as little as 90 minutes of MI. Previous studies have reported that in general, higher "doses" of MI tend to be associated with larger effects, ${ }^{36,38}$ so the results of the present study are promising in that they suggest that even brief doses of MI are associated with medium effects on adherence. It is also noteworthy that the superior net improvement in ICS adherence in the MI group was observed in comparison to control condition that likely approximates "standard or optimal" care rather than "usual" care. In this study, the control groups were all treated by experienced pulmonologists who treat asthmatics according to guideline recommendations: standard practice at our hospital-based asthma clinic is to give patients a prescription for ICS + reliever medication as needed, and/or an action plan for exacerbations, and/or referral to in-house asthma education. As such, it is possible that the "strength" of the control group in this study may have weakened some of the effects associated with MI. Nonetheless, the fact that MI was delivered as a brief intervention also suggests that it may have important cost-effectiveness for improvement in ICS adherence and ultimately asthma outcomes.

Although there were no significant differences in relation to changes in12-month mean asthma control and overall quality of life levels, medium ESs (ranging from $d=0.31$ to 0.36 ) in favor of the intervention were observed on the Activity Limitation, Environmental Impact, and Emotion subscales of the AQLQ among patients who completed the intervention. This suggests that MI and any changes in adherence may more readily impact functional measures of asthma in these domains, and that more time may be needed to observe larger effects on asthma control. The failure to observe any group differences in relation to changes in asthma control may also be due to the relatively small MI vs control difference in adherence change. To our knowledge, this is the first study to assess the impact of MI on changes in asthma control and quality of life levels. However, results suggest that in modest doses, MI may impact some clinical measures of asthma more than others, eg, those related to functional status and quality of life.

Interestingly, the largest ESs observed in association with the intervention were in relation to changes in 12-month asthma self-efficacy in both ITT $(d=0.43)$ and completer 
$(d=0.53)$ analyses. This is not surprising as one of the key goals of MI in clinical settings is to increase self-efficacy or confidence in patients' ability to change their behavior or engage in better self-management of their disease. This result is also reassuring as it provides evidence for improved selfefficacy as a potential moderator of the intervention's impact on adherence and asthma outcomes. However, the fact the self-efficacy effect was larger than the adherence effects suggests that MI may have stronger impacts on confidence that take longer to translate into actual behavior change. To our knowledge, this is the first study to assess the impact of MI on changes in asthma-specific self-efficacy. However, the results are highly consistent with previous studies showing the impact of MI on measures of self-efficacy ${ }^{65,66}$ and points to a specific target of intervention among poorly controlled, nonadherent asthmatics. Although we did not assess them or attempt to intervene upon them in this study, other psychological explanations for low adherence have been reported in the literature including depression, cognitive impairments, and distorted illness and/or treatment beliefs. ${ }^{66-68}$ Future studies could assess the extent to which MI could also improve other psychological variables.

\section{Study limitations and strengths}

The present study has some limitations that warrant caution in the interpretation of findings. First, our primary measure of adherence used pharmacy refill data, which does not assess actual medication consumption. However, assessing pharmacy refill data is considered to be one of the most reliable methods of assessing adherence relative to self-reports, dose/ pill counters, and biological assessments, ${ }^{69}$ the former two of which have been shown to be subject to reporting bias and/or tampering when the participant is aware that they are being monitored. ${ }^{67}$ Second, patients were recruited from a single tertiary care center, so results may not generalize to asthmatics treated in primary care or community samples. However, a sample of moderate-severe asthmatics was deliberately targeted given their relatively greater needs for ICS medication and the importance of regular adherence to achieve control. Third, recruitment rates were surprisingly low at $23 \%$. The reasons for such low rates of recruitment are not known, and ethically, we were not permitted to ask patients their reasons for nonparticipation. One of the challenges associated with ICS adherence among asthmatics is the accuracy of their estimates of their levels of control. This project was presented as one aimed at helping patients improve their levels of asthma control. It is therefore possible that patients' overestimated their levels of control and did not perceive a need or see a potential benefit to participating. Unfortunately, we did not assess patients' perceptions of their levels of control so we cannot confirm this hypothesis directly. However, patients' poor overall control levels (ie, ACQ $=1.9$ ) and surprisingly low baseline rates adherence (ie, $<30 \%$ ) indicates a disconnection between actual control levels and medication use, suggesting that patients may be underestimating their levels of asthma control. It should also be noted that we also recruited patients who were highly $(<50 \%)$ adherent, which is lower than many reported definitions of nonadherence, which is around $<80 \%{ }^{67,70}$ This may have also impacted our recruitment rates. Fourth, measures of asthma control and quality of life were based on self-reports, which may be subject to recall bias. However, we also collected objective measures of lung function, which were incorporated into ACQ scores. Finally, as mentioned earlier, the results of this study likely failed to reach statistical significance due to a low sample size and lack of power. However, this aspect of the design was intentional and was instituted to comply with the recommendation of ORBIT to use a phasic approach to behavioral intervention design. In line with this recommendation, we conducted a Phase II trial to determine the size of the effect associated with a 90-minute "dose" of MI and the acceptability of the intervention to patients. The medium $\operatorname{ES}(d=0.20)$ and high patient acceptability (postintervention ratings for pertinence, usefulness, enjoyableness, feasibility, and likely to change adherence behavior all $\geq 8.9 / 10$ ) suggest that a full randomized controlled trial is warranted, and this pilot data will permit an accurate estimation of the sample size needed to reach statistical significance on both primary and secondary outcomes.

Despite these limitations, this study also has several important strengths. First, it is the first adherence intervention among adult asthmatics to test the feasibility of a theory-driven intervention that was specifically designed to promote behavior change. Second, it measured baseline and follow-up adherence using a well-validated, objective measure (\% pharmacy refills), which reduces the likelihood of recall bias (self-reports) and subject tampering (dose counters and biological assessments) and improves the overall reliability of the measure. Third, we included patients who were nonadherent to ICS medication, ensuring that only participants who had behavior change were intervened upon. This is important because previous studies that have failed to find a significant effect for MI may have failed because they attempted to intervene upon patients who did not exhibit the "problem" behavior in the first place. ${ }^{71}$ For example, Solomon et $\mathrm{al}^{71}$ examined the efficacy of MI to 
improve adherence among newly diagnosed osteoporosis patients and failed to find a significant effect. However, they enrolled any patient who received a new prescription, regardless of their typical level of adherence. As such, it is possible that some patients were enrolled in the trial despite having no problems with adherence. Even though one might expect this to be evenly distributed between the groups (due to randomization), it may have ultimately diluted any treatment effect (ie, reduced power) and contributed to the nonsignificant findings observed. ${ }^{72}$ Fourth, we employed a manualized intervention (with some degree of flexibility to be consistent with the spirit of MI), which increases the reproducibility of the findings. Fifth, we used an experienced ( $>10$ years) clinical psychologist who received extensive MI training with regular supervision and feedback to administer the intervention. This, coupled with an assessment of treatment fidelity (which was judged to have been delivered at the expert level by an experienced, trained evaluator) ensures the integrity of intervention. Sixth, we included outcomes at the behavioral (adherence), clinical (asthma control, quality of life), and process (asthma self-efficacy) level. In any behavioral intervention that targets behavior change, it is critical to include the behavior of interest as the primary outcome. Finally, analyses were adjusted for several important covariates (including sex, age, ICS dose [as an indicator of asthma severity], and smoking), and included a follow-up period long enough to estimate the 12-month efficacy of the intervention, which is critical to determine the long-term maintenance of behavior change.

\section{Conclusion}

The results of this pilot RCT suggest that a brief (90 minutes) intervention using MI to enhance intrinsic motivation and self-efficacy to improve medication (ICS) adherence can produce improvements in adherence behavior in poorly controlled, nonadherent asthmatics at 6 months that are maintained at 1 year, and is well accepted by patients. This trial provides evidence of the need for a fully powered clinical trial to assess the impact of MI on both medication adherence and relevant asthma outcomes (eg, asthma control levels, health service use, asthma-related quality of life).

\section{Acknowledgments}

Funding support for this study was provided by an unrestricted, investigator-initiated grant from GlaxoSmithKline and salary awards from the Fonds de la recherche du Québec - Santé (FRQS) (KLL and SLB) and the Canadian Institutes of Health Research (KLL and SLB). Scholarship support was also provided by the FRQS (GM). The authors thank Dr Jean-Luc Malo, MD; Guillaume Lacoste, BA; Sandra Pelaez, PhD; Nicole Rutamucero, MSc; and Melanie Beland, MSc, for their invaluable assistance with data collection; Wendy Smith, $\mathrm{PhD}$, for conducting the MI intervention; and Catherine Laurin, $\mathrm{PhD}$, for conducting intervention integrity evaluations.

\section{Disclosure}

KLL has received professional consultation and presentation fees from Merck, Boehringer Ingelheim, Takeda, AbbVie and Kataka for the development and delivery of training programs related to MI. SLB has received professional consultation and presentation fees from Merck and Kataka for the development and delivery of training programs related to MI. The other authors report no conflicts of interest in this work.

\section{References}

1. Agence de la santé publique du Canada. La vie et le souffle: Les maladies respiratoires au Canada [Life and breath: respiratory diseases in Canada]; 2007. Retiré le 1 avril 2011, de. Available from: http:// www.phac-aspc.gc.ca/publicat/2007/lbrdc-vsmrc/index-fra.php. French.

2. Center for Disease Control. Asthma in the US: growing every year. $C D C$ Vital Signs. 2011:1-4. Available from: http://www.cdc.gov/VitalSigns/ pdf/2011-05-vitalsigns.pdf. Accessed June 24, 2013.

3. Chapman KR, Boulet LP, FitzGerald MJ, McIvor RA, Zimmerman S. Patient factors associated with suboptimal asthma control in Canada: results from the reality of asthma control study. Am J Respir Crit Care Med. 2005;172:A678.

4. Chapman KR, Ernst P, Grenville A, Dewland P, Zimmerman S. Control of asthma in Canada: failure to achieve guideline targets. Can Respir J. 2001;8(suppl A):35A-40A.

5. Global Initiative for Asthma. Global Strategy for Asthma Management and Prevention: NIH Publication No 02-3659; 2005. Available from http://www.ginasthma.org/local/uploads/files/GINA_Report_2014_ Aug12.pdf. Accessed September 29, 2014.

6. Bateman ED, Boushey HA, Bousquet J, et al. Can guideline-defined asthma control be achieved? The gaining optimal asthma control study. Am J Respir Crit Care Med. 2004;170(8):836-844.

7. Boulet LP, Bai TR, Becker A, et al. What is new since the last (1999) Canadian Asthma Consensus Guidelines? Can Respir J. 2001;8(A): 5A-27A.

8. Bender B, Milgrom H, Rand C. Nonadherence in asthmatic patients: is there a solution to the problem? Ann Allergy Asthma Immunol. 1997;79: 177-185.

9. Bender BG, Long A, Parasuraman B, Tran ZV. Factors influencing patient decisions about the use of asthma controller medication. Ann Allergy Asthma Immunol. 2007;98:322-328.

10. Schmaling KB, Hernandez DV, Giardino ND, editors. Provider and patient adherence with asthma evaluation and treatment. In: Brown ES, editor. Asthma: Social and Psychological Factors and Psychosomatic Syndromes. Advances in Psychosomatic Medicine No. 24. Basel, Switzerland: Karger; 2003:98-114.

11. Horne R. Compliance, adherence, and concordance: implications for asthma treatment. Chest. 2006;130(1 suppl):65S-72S.

12. Ivanova JI, Birnbaum HG, Hsieh M, et al. Adherence to inhaled corticosteroid use and local adverse events in persistent asthma. $\mathrm{Am} J$ Manag Care. 2008;14(12):801-809.

13. Laforest L, El Hasnaoui A, Pribil C, et al. Asthma patients' self-reported behaviours toward inhaled corticosteroids. Respir Med. 2009;103(9): 1366-1375. 
14. Menckeberg TT, Bouvy ML, Bracke M, et al. Beliefs about medicines predict refill adherence to inhaled corticosteroids. J Psychosom Res. 2008;64(1):47-54.

15. Bartlett SJ, Lukk P, Butz A, Lampros-Klein F, Rand CS. Enhancing medication adherence among inner-city children with asthma: results from pilot studies. J Asthma. 2002;39:47-54.

16. Burgess SW, Sly PD, Devadason SG. Providing feedback on adherence increases use of preventive medication by asthmatic children. J Asthma. 2010;47:198-201.

17. Burkhart PV, Rayens MK, Oakley MG, Abshire DA, Zhang M. Testing an intervention to promote children's adherence to asthma selfmanagement. J Nurs Scholarsh. 2007;39-40:133-140.

18. da Costa IG, Rapoff MA, Lemanek K, Goldstein GL. Improving adherence to medication regimens for children with asthma and its effect on clinical outcome. J Appl Behav Anal. 1997;30:687-691.

19. Wilson SR, Strub P, Buist AS, et al. Shared treatment decision making improves adherence and outcomes in poorly controlled asthma. Am J Respir Crit Care Med. 2010;181:566-577.

20. Bender BG, Apter A, Bogen DK, et al. Test of an interactive voice response intervention to improve adherence to controller medications in adults with asthma. J Am Board Fam Med. 2010;23:159-165.

21. Berg J, Dunbar-Jacob J, Sereika SM. An evaluation of a self-management program for adults with asthma. Clin Nurs Res. 1997;6:225-238.

22. Bailey WC, Richards JMJ, Brooks CM, Soong SJ, Windsor RA, Manzella BA. A randomized trial to improve self-management practices of adults with asthma. Arch Intern Med. 1990;150:1664-1668.

23. Schaffer SD, Tian L. Promoting adherence: effects of theory-based asthma education. Clin Nurs Res. 2004;13(1):69-89.

24. Windsor RA, Bailey WC, Richards JMJ, Manzella BA, Soong SJ, Brooks M. Evaluation of the efficacy and cost effectiveness of health education methods to increase medication adherence among adults with asthma. Am J Public Health. 1990;80:1519-1521.

25. Chatkin JM, Blanco DC, Scaglia N, Wagner MB, Fritscher CC. Impact of a low-cost and simple intervention in enhancing treatment adherence in a Brazilian asthma sample. J Asthma. 2006;43:263-266.

26. Gallefoss F, Bakke PS. How does patient education and self-management among asthmatics and patients with chronic obstructive pulmonary disease affect medication adherence? Am J Respir Crit Care Med. 1999; 160:2000-2005.

27. Janson SL, McGrath KW, Covington JK, Cheng SC, Boushey HA Individualized asthma self-management improves medication adherence and markers of asthma control. J Allergy Clin Immunol. 2009; 123(4):840-846

28. Put C, van den Bergh O, Lemaigre V, Demedts M, Verleden G. Evaluation of an individualised asthma programme directed at behavioural change. Eur Respir J. 2003;21(1):109-115.

29. Mehuys E, Van Bortel L, De Bolle L, et al. Effectiveness of pharmacist intervention for asthma control improvement. Eur Respir J. 2008 31:790-799.

30. Altman DG, Schulz KF, Moher D, et al. The revised CONSORT statement for reporting randomized trials: explanation and elaboration. Ann Intern Med. 2001;134:663-694.

31. Kolbe J, Vamos M, Fergusson W, Elkind G, Garrett J. Differential influences on asthma self-management knowledge and selfmanagement behavior in acute severe asthma. Chest. 1996;110(6): 1463-1468.

32. Kolbe J, Vamos M, James F, Elkind G, Garrett J. Assessment of practical knowledge of self-management of acute asthma. Chest. 1996;109(1) 86-90.

33. Julius RJ, Novitsky MA Jr, Dubin WR. Medication adherence: a review of the literature and implications for clinical practice. J Psychiatr Pract. 2009;15:34-44.

34. Rollnick S, Miller WR, Butler CC. Motivational Interviewing in Health Care: Helping Patients Change Behavior. New York, NY: Gilford Press; 2008.

35. Miller WR, Rollnick S. Motivational Interviewing: Preparing People for Change. New York, NY: Guilford Press; 2002.
36. Rubak S, Sandboek A, Lauritzen T, Christensen B. Motivational interviewing: a systematic review and meta-analysis. Br J Gen Pract. 2005; 55:305-312.

37. Hettema J, Steele J, Miller WR. Motivational interviewing. Annu Rev Clin Psychol. 2005;1:91-111.

38. Lundahl B, Moleni T, Burke BL, et al. Motivational interviewing in medical care settings: A systematic review and meta-analysis of randomized controlled trials. Patient Educ Couns. 2013;93(2):157-168.

39. American Thoracic Society. Standardization of spirometry, 1994 update. Am J Respir Crit Care Med. 1995;152(3):1107-1136.

40. Czajkowski S. Available from: http://www.nhlbi.nih.gov/resources/ obesity/trials/orbit.htm. 2009. Accessed March 20, 2014.

41. Moher D, Jones A, Lepage L. Use of the CONSORT statement and quality of reports of randomized trials: a comparative before-and-after evaluation. JAMA. 2001;285(15):1992-1995.

42. Peláez S, Aulls MW, Lacoste G, Bacon SL, Lavoie KL. Potential interventions to enhance adherence to asthma treatment. Int J Qual Methods. 2012;11(5):763.

43. DiClemente CC, Velasquz MM. Motivational interviewing and the stages of change. In: Miller WR, Rollnick S, editors. Motivational Interviewing: Preparing People for Change. 2nd ed. New York, NY: Gilford Press; 2002:201-216.

44. Moyers TB, Martin T, Manuel JK, Hendrickson SM, Miller WR. Assessing competence in the use of motivational interviewing. J Subst Abuse Treat. 2005;28:19-26.

45. Morisky E, Green LW, Levine DM. Concurrent and predictive validity of a self-reported measure of medication adherence. Med Care. 1986;2: 759-782.

46. Bailey WC, Kohler CL, Richards JM Jr, et al. Asthma self-management: do patient education programs always have an impact? Arch Intern Med. 1999; 159(20):2422-2428.

47. Juniper EF, O’Byrne PM, Guyatt GH, Ferrie PJ, King DR. Development and validation of a questionnaire to measure asthma control. Eur Respir J. 1999;14(4):902-907.

48. Nathan RA, Sorkness CA, Kosinski M, et al. Development of the asthma control test: a survey for assessing asthma control. J Allergy Clin Immunol. 2004;113(1):59.

49. GINA Dissemination Committee. Dissemination and Implementation of Asthma Guidelines. Global Initiative for Asthma; 2003.

50. Juniper EF, O’Byrne PM, Ferrie PJ, King DR, Roberts JN. Measuring asthma control. Clinic questionnaire or daily diary? Am J Respir Crit Care Med. 2000;162(4 pt 1):1330-1334.

51. Juniper EF, Bousquet J, Abetz L, Bateman ED. The Goal Committee. Identifying 'well-controlled' and 'not well-controlled' asthma using the Asthma Control Questionnaire. Respir Med. 2006;100: 616-621.

52. Juniper EF, Stahl E, Mork AC, Svensson K. Minimal important difference for the asthma control questionnaire. Eur Respir J. 2004;24:460s.

53. Schatz M, Kosinski M, Yarlas AS, Hanlon J, Watson ME, Jhingran P. The minimally important difference of the Asthma Control Test. J Allergy Clin Immunol. 2009;124:719-723.

54. Juniper EF, Guyatt GH, Ferrie PJ, Griffith LE. Measuring quality of life in asthma. Am Rev Respir Dis. 1993;147(4):832-838.

55. Leidy NK, Coughlin C. Psychometric performance of the Asthma Quality of Life Questionnaire in a US sample. Qual Life Res. 1998;7(2): $127-134$.

56. Juniper EF, Guyatt GH, Willan A, Griffith LE. Determining a minimal important change in a disease-specific Quality of Life Questionnaire. $J$ Clin Epidemiol. 1994;47(1):81-87.

57. Tobin DL, Wigal JK, Winder JA, Holroyd KA, Creer TL. The "asthma self-efficacy scale". Ann Allergy. 1987;59(4):273-277.

58. Lavoie KL, Bouchard A, Joseph M, Campbell TS, Favreau H, Bacon SL. Association of asthma self-efficacy to asthma control and quality of life. Ann Behav Med. 2008;36:100-106.

59. Bacon SL, Bouchard A, Loucks E, Lavoie KL. Is socio-economic status associated with worse asthma morbidity in a Canadian sample of adult asthmatics? Respiratory Research. 2009;10:125. 
60. Barzi F, Woodward M. Imputations of missing values in practice: results from imputations of serum cholesterol in 28 cohort studies. $\mathrm{Am}$ J Epidemiol. 2004;160(1):34-45.

61. Rubin DB. Multiple Imputation for Nonresponse in Surveys. Vol. 1-288. New York: John Wiley \& Sons; 1987.

62. Cohen J. Statistical Power Analysis for the Behavioral Sciences. Hillsdale, NJ: Erlbaum; 1988.

63. Schmaling KB, Blume AW, Afari N. A randomized controlled pilot study of motivational interviewing to change attitudes about adherence to medications for asthma. J Clin Psychol Med Settings. 2001;8: $167-172$.

64. Riekert KA, Borrelli B, Bilderback A, Rand CS. The development of a motivational interviewing intervention to promote medication adherence among inner-city, African-American adolescents with asthma. Patient Educ Couns. 2010;82:117-122.

65. Chariyeva Z, Golin CE, Earp JA, Maman S, Suchindran C, Zimmer C. The role of self-efficacy and motivation to explain the effect of motivational interviewing time on changes in risky sexual behavior among people living with HIV: a mediation analysis. AIDS Behav. 2013;17:813-823.

66. Chen SM, Creedy D, Lin HS, Wollin J. Effects of motivational interviewing intervention on self-management, psychological and glycemic outcomes in type 2 diabetes: a randomized controlled trial. Int J Nurs Stud. 2012;49:637-644.
67. Osterberg L, Blaschke T. Adherence to medication. NEngl J Med. 2005; 353(5):487-497.

68. DiMatteo MR, Lepper HS, Croghan TW. Depression is a risk factor for noncompliance with medical treatment: meta-analysis of the effects of anxiety and depression on patient adherence. Arch Intern Med. 2000; 160(14):2101-2107.

69. Gamble J, Stevenson M, McClean E, Heaney LG. The prevalence of nonadherence in difficult asthma. Am J Respir Crit Care Med. 2009;180: $817-822$.

70. Brown MT, Bussell JK. Medication adherence: WHO cares? Mayo Clin Proc. 2011;86:304-314.

71. Solomon DH, Iversen MD, Avorn J, et al. Osteoporosis Telephonic Intervention to Improve Medication Regimen Adherence: A Large, Pragmatic, Randomized Controlled Trial. Arch Intern Med. 2012;172:477-483.

72. Lavoie KL, Campbell TS, Bacon SL. Does motivational interviewing improve medication adherence? Arch Int Med. 2012;172:1351-1352. 


\section{Supplementary material}

Table SI Participants' characteristics at baseline

\begin{tabular}{|c|c|c|c|}
\hline & Completers $(n=40)$ & Withdrawals $(n=14)$ & $P$ \\
\hline \multicolumn{4}{|l|}{ Sociodemographics } \\
\hline Age, years & $5 I(15)$ & $50(17)$ & 0.83 \\
\hline Men, n (\%) & $14(35)$ & $7(50)$ & 0.32 \\
\hline Cohabitation (yes), n (\%) & $24(60)$ & $6(43)$ & 0.27 \\
\hline Employed (yes), n (\%) & $18(45)$ & $9(65)$ & 0.21 \\
\hline Education level ( $\geq 12$ years), $n(\%)$ & $21(52)$ & $9(64)$ & 0.44 \\
\hline \multicolumn{4}{|l|}{ Clinical and functional profile } \\
\hline Refill: $\beta 2$ LA + ICS or ICS alone, $\%$ & $15(38)$ & $6(33)$ & 0.72 \\
\hline Refill: combined $\beta 2 L A+I C S, \%$ & $25(62)$ & $8(57)$ & \\
\hline Total dose of combined $\beta 2 \mathrm{LA}$ and/or ICS, mg & $739(46 I)$ & $675(6 \mid 8)$ & 0.68 \\
\hline Anti-leukotrienes, n (\%) & II (27) & I (7) & 0.15 \\
\hline Anti-rhinitics, n (\%) & $13(32)$ & $6(43)$ & 0.53 \\
\hline $\mathrm{FEV}_{1}, \mathrm{~L}$ & $2.0(0.7)$ & $2.2(1.1)$ & 0.51 \\
\hline $\mathrm{FEV}_{1}, \%$ predicted & $67(15)$ & $74(39)$ & 0.51 \\
\hline FVC, L & $2.4(1.1)$ & $2.7(1.3)$ & 0.53 \\
\hline FVC, \% predicted & $67(17)$ & $77(54)$ & 0.47 \\
\hline $\mathrm{FEV}_{1} / \mathrm{FVC}, \%$ predicted & $103(17)$ & $102(18)$ & 0.78 \\
\hline Atopic, n (\%) & $33(82)$ & $10(72)$ & 0.45 \\
\hline Current smoker (yes), n (\%) & $4(10)$ & $3(21)$ & 0.36 \\
\hline $\mathrm{BMI}, \mathrm{kg} \cdot \mathrm{m}^{-2}$ & $31(8)$ & $29(6)$ & 0.53 \\
\hline Waist circumference, $\mathrm{cm}$ & $95(16)$ & $95(10)$ & 0.98 \\
\hline Asthma duration, years & $25(20)$ & $21(12)$ & 0.44 \\
\hline Bronchodilator use, \# last week & $6(8)$ & $12(19)$ & 0.25 \\
\hline Bronchodilator use, \# per puff & $1.5(0.5)$ & $1.4(0.5)$ & 0.30 \\
\hline ACT scores (5-25) & $17(4)$ & $18(3)$ & 0.22 \\
\hline ACQ scores $(0-6)$ & $1.9(0.9)$ & $2.0(1.3)$ & 0.61 \\
\hline ASES total scores $(I-5)$ & $2.6(0.1)$ & $2.6(0.2)$ & 0.91 \\
\hline Morisky scores (5-30) & $22(5)$ & $22(4)$ & 0.93 \\
\hline \multicolumn{4}{|l|}{ AQLQ scores (I-7) } \\
\hline Total & $4.9(0.9)$ & $4.9(1.2)$ & 0.94 \\
\hline Activity limitations & $4.5(1.2)$ & $4.6(1.4)$ & 0.75 \\
\hline Symptoms & $5.0(0.9)$ & $4.9(1.2)$ & 0.78 \\
\hline Emotional function & $5.4(1.2)$ & $5.5(1.3)$ & 0.77 \\
\hline Exposure & 4.7 (I.4) & $4.7(1.4)$ & 0.96 \\
\hline
\end{tabular}

Notes: Data are expressed as mean (SD) or $n$ (\%).

Abbreviations: MI, motivational interviewing; $\beta 2 \mathrm{LA}$, long-acting beta2-agonists; ICS, inhaled corticosteroid; FEV , forced expiratory volume in I second; FVC, forced vital capacity; BMI, body mass index; AQLQ, asthma quality-of-life questionnaire; ACT, Asthma Control Test; ACQ, Asthma Control Questionnaire; ASES, Asthma Self-Efficacy Scale.

\section{Publish your work in this journal}

Patient Preference and Adherence is an international, peer-reviewed, open access journal that focuses on the growing importance of patient preference and adherence throughout the therapeutic continuum. Patient satisfaction, acceptability, quality of life, compliance, persistence and their role in developing new therapeutic modalities and compounds to optimize

\section{Dovepress}

clinical outcomes for existing disease states are major areas of interest for the journal. This journal has been accepted for indexing on PubMed Central. The manuscript management system is completely online and includes a very quick and fair peer-review system, which is all easy to use. Visit http://www. dovepress.com/testimonials.php to read real quotes from published authors. 